\title{
Credibility Assessment of Twitter Data using Machine Learning Algorithms

\author{
${ }^{1}$ T.Prashanthi, ${ }^{2}$ T. Rajesh \\ ${ }^{1,2}$ Department of Computer Science and Engineering, G. Narayanamma Institute of Technology \& Science for \\ Women, Hyderabad \\ Email: prashanthi.talla18@gmail.com,rajesht531@gmail.com
}

Received: 06th April 2020, Accepted: 05th May 2020, Published: 30th June 2020

\begin{abstract}
The augmenting of the Internet has brought about data dispersal that turns out to be progressively moderate, implying that clients can get to data from anyplace on the planet utilizing PCs and advanced mobile phones. Data Credibility on Twitter has been a theme of enthusiasm among analysts in the fields of both PC and sociologies. Twitter has made it progressively conceivable to offer close ongoing exchange of data in a very financially savvy way. It is presently being utilized as a wellspring of news among a wide cluster of clients around the world.

Data validity has gone under investigation, particularly in interpersonal organizations that are presently being utilized effectively as first wellsprings of data. An ongoing substance credibility assessment system named CredFinder is fit for estimating the dependability of data through client investigation and substance examination. PageRank-like credibility propagation technique is used to evaluate validity data on twitter. No Machine Learning calculations are utilized. The framework proposes another credibility examination framework for surveying data validity on Twitter to anticipate the expansion of phony or malignant data. The proposed framework comprises of four coordinated segments: a notoriety based part, a believability classifier motor, a client experience segment, and a component positioning calculation. The segments work together in an algorithmic structure to break down and survey the credibility of Twitter tweets and clients.
\end{abstract}

\section{Keywords}

Credibility, Notoriety, Believability, Client Experience, Component Positioning.

\section{Introduction}

Online informal communities, for example, Twitter, have become profoundly main stream in the 21 st century, as the quantities of clients who are utilizing them on consistent schedule bear witness to. The way that clients are permitted to convey what needs be with practically zero control is likewise another: exceptionally alluring part of these stages.[1] Research on data validity is in this way the best answer for the issue of how to evaluate the credibility of data and maybe moderate the spread of falsehood. Right now, specialists have utilized different philosophies in concentrates on data validity. Some of them believe the issue to be one of arrangement that ought to be explained in a mechanized manner utilizing AI or chart based calculations. Others see it as a subjective issue requiring human-driven confirmation. A few scientists have ventured to such an extreme as to make frameworks evaluate credibility and progressively. Such frameworks incorporate Tweetcred and TwitterTrails. There has additionally been a huge measure of research concentrated on this theme in instances of highsway occasions, for example, tremors, floods, and political developments.

\section{Methodology}

1. Pre-handling of the information like Removing all accentuations, images, numbers, Removing Stop Words and so on is done since the crude information having extremity is profoundly helpless to irregularity and excess.

2. Highlight Extraction is done to remove the viewpoints from the handled information. These perspectives or highlight vectors are utilized to process the positive or negative tweets in a sentence which is helpful for deciding the assessment of the people.

3. Training is done using supervised learning techniques to make it easier for prediction of unknown tweets.

4. Decision Tree Classifier and Feature Ranking Algorithm is used for the classification purpose. 


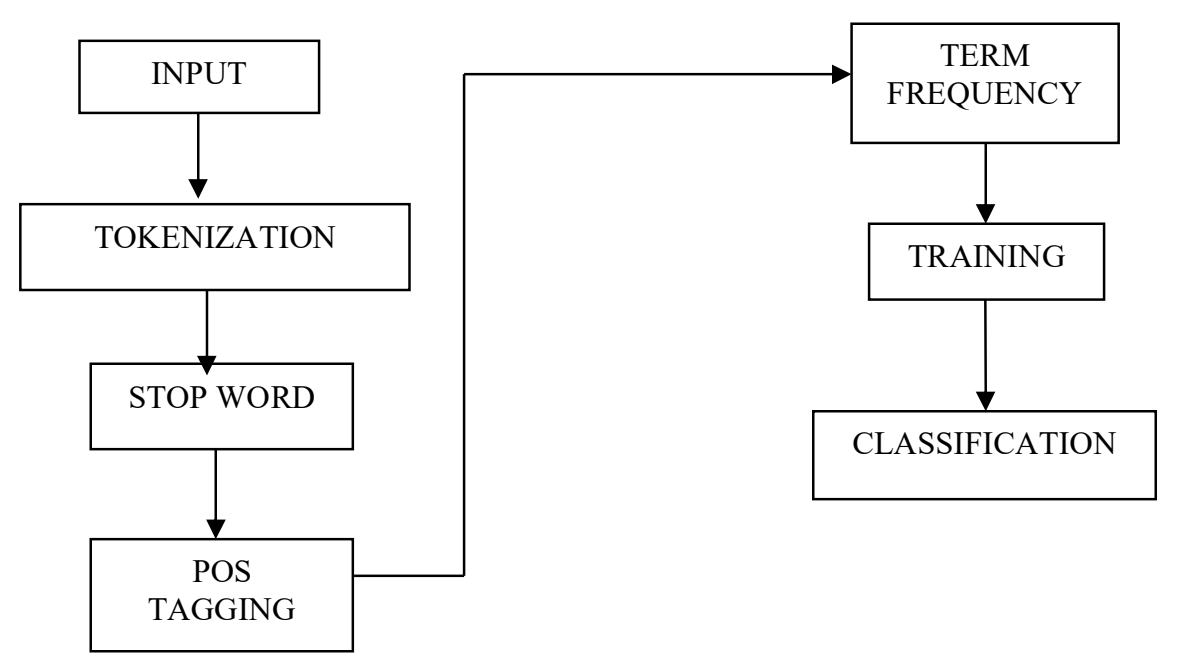

\section{Computing the Sentiment History}

Fig 1: Process used in Proposed System

The wistfulness of a client impacts their decisions of tweet validity as for an occasion or subject, particularly when the client is slanted positively or ominously toward certain organizations or gatherings. A few clients have purpose behind dispersing data that might be viewed as deluding and can add to turmoil.[2] Supposition characterizes the components that influence social connections, mental conditions of clients, and their direction. Assessment likewise includes an investigation of why a client confides in a trustee or not. In an investigation on computation of the quantity of positive and negative words in a message, in view of a predefined "assumption words" list, scientists found that the least solid messages are related with negative gettogethers and contain solid negative notion words and feelings [6].

For each customer $\mathrm{u}_{\mathrm{i}} \in \mathrm{U}$, we figure an inclination score(signified by $\Delta \mathrm{u}$ ) considering assessment of his past tweets, using the going with condition:

$$
\Delta_{u_{t}}=\frac{\sum T_{u_{t}}^{+}}{\sum T_{u_{t}}^{+}+\sum\left|T_{u_{t}}^{-}\right|}
$$

where, $\mathrm{T}^{+}$is a customer's sure tweets and $\mathrm{T}^{-}$is a customer's negative tweets, dictated by the Arabic inclination examination count SAMAR[4].

\section{Estimation of User Popularity Score}

The Score which is an extent of customer commonness, is obtained from an essential calculating explanation that empowers the formation of crucial information concerning casual networks, considering a numerical worth. Estimations can be joined to describe a situating measure. This measure can be explained as a count that delineates standards suitable for situating each customer on the framework as for his reputation. Accept that we have the $U$ set of customers who have more than one tweet on a given subject peP. Given a great deal of tweets (connoted by T), we figure the tweets of each customer $t_{u}$ over T. Thus, the fundamental estimation of customer activity is according to the accompanying:

$$
\mathrm{I}_{\mathrm{P}}\left(\mathrm{u}_{\mathrm{i}}\right)=\left\{\sum_{u \in U} \operatorname{ceP} t^{\mathrm{p}} \mathrm{u}_{\mathrm{i}} /|\mathrm{T}| \text {, if } \mathrm{t}_{\mathrm{ui}} \epsilon|\mathrm{T}|\right.
$$

0 , otherwise

Taking into account our discernments, we consider re-tweets, top picks, and notification to be the best pointers from a quantitative perspective. This deduces a tweet that has been re-tweeted normally is seen as engaging the peruser. Before long, the most fundamental pointers are abstract. One authentic model is the association between the peruser and the tweeter of the tweet. Such examinations are an epic impediment to the significance of a customer's dominance. We at first figure the social reputation $\vartheta$ of $u \in U$ on a given point $p \in P$ using the going with condition:

$$
\Theta_{\mathrm{p}} \in \mathrm{P}\left(\mathrm{u}_{\mathrm{i}}\right)=\log \left(\operatorname{NoFlw}\left(\mathrm{u}_{\mathrm{i}}\right)\right) / \max (\log (\operatorname{NoFlw}(\mathrm{U}, \mathrm{p}))
$$

By then, we figure the event duty of customer $u \in U$ on a given subject $p \in P$ utilizing the amount of top picks $\varphi$, the amount of re-tweets $\mathrm{v}$ and the amount of notification $\psi$ concerning topic $\mathrm{p}$ as showed up in conditions 4,5 and 6 . 
$\phi p \epsilon \mathrm{P}\left(\mathrm{u}_{\mathrm{i}}\right)=\log \left(\operatorname{NoUFav}\left(\mathrm{u}_{\mathrm{i}}\right)\right) / \max (\log (\operatorname{NoUFavU}, \mathrm{p}))$

The amount of re-tweets of his tweets on a comparable point $\mathrm{p}$ is resolved as showed up in condition 5 .

$\operatorname{\omega vp\epsilon P}\left(\mathrm{u}_{\mathrm{i}}\right)=\log \left(\operatorname{NoRT}\left(\mathrm{u}_{\mathrm{i}}\right)\right) / \max (\log (\operatorname{NoRT}(\mathrm{U}, \mathrm{p}))$

The events the customer has been referenced in others' tweets on a comparable given subject $\mathrm{p}$ is resolved as showed up in condition 6 .

$\psi p \in \mathrm{P}\left(\mathrm{u}_{\mathrm{i}}\right)=\log \left(\mathrm{NoM} \mathrm{e}\left(\mathrm{u}_{\mathrm{i}}\right)\right) / \max (\log (\mathrm{NoM} \mathrm{e}(\mathrm{U}, \mathrm{p}))$

The event responsibility of customer $u$ on subject $\mathrm{p}$ is settled as an immediate mix of the recently referenced given subject $\mathrm{p} \epsilon \mathrm{P}$

$\operatorname{EE}^{\mathrm{p} \epsilon \mathrm{P}}\left(\mathrm{u}_{\mathrm{i}}\right)=\phi^{\mathrm{p}}\left(\mathrm{u}_{\mathrm{i}}\right)+v^{\mathrm{p}}\left(\mathrm{u}_{\mathrm{i}}\right)+\psi^{\mathrm{p}}\left(\mathrm{u}_{\mathrm{i}}\right)$

For a given subject $\mathrm{p} \epsilon \mathrm{P}$, customer sway, implied by $\omega(\mathrm{u})$ can be figured as seeks after:

$\mathrm{w}^{\mathrm{peP}}\left(\mathrm{u}_{\mathrm{i}}\right)=\partial^{\mathrm{p}}\left(\mathrm{u}_{\mathrm{i}}\right)+\mathrm{EE}^{\mathrm{p}}\left(\mathrm{u}_{\mathrm{i}}\right)+\mathrm{I}^{\mathrm{P}}\left(\mathrm{u}_{\mathrm{i}}\right) / \log (\square)$

We use to demonstrate the amount of customers considered concerning topic $\mathrm{p}$ as showed up in the formula above.

The last development is to rank the customers according to their reputations, which can be resolved as seeks after:

$\mathrm{R}^{\mathrm{p}}\left(\mathrm{u}_{\mathrm{i}}\right)=\Delta \mathrm{u}_{\mathrm{i}} *\left(\mathrm{w}^{\mathrm{p}\left(\mathrm{u}_{\mathrm{i}}\right)}\right)$

Clients for whom the estimation of is lower than 0.1 for clients are viewed as inconsistent/non-solid sources.

The clients with the most note worthy priority values are viewed as the most confided in sources on a given point, while the clients with the least need esteems are viewed as the least trusted. The positioned rundown of clients is a contribution to the following strategy.

Users for whom the value of $\mathrm{R}^{\mathrm{p}}\left(\mathrm{u}_{\mathrm{i}}\right)$ is lower than 0.1 forusers are considered to be unreliable/non- credible sources.

The users with the highest priority $\mathrm{R}^{\mathrm{P}}\left(\mathrm{u}_{\mathrm{i}}\right)$ values are considered to be the most trusted sources on a given topic, while the users with the lowest priority values are considered to be the least trusted. The ranked list of users is an input to the next technique.

\section{Feature Ranking Algorithm}

In Feature Ranking Algorithm the extricated highlights ought to be weighted before figuring the appraisal of a given tweet, client, or point, in view of impact of the highlights on the last judgment of validity. In my examination on believability of social web content, I have reasoned that the quantity of devotees is the most significant component, trailed by the quantity of message URLs, re-tweets, and client makes reference.

$$
\Lambda=\begin{array}{cccc}
1 & f_{12} & \cdots & f_{1 n} \\
f_{21} & 1 & \cdots & f_{2 n} \\
\vdots & \vdots & \ddots & \vdots \\
f_{n 1} & f_{n 2} & \cdots & 1
\end{array}
$$

The least persuasive elements among those considered were finished up to be the time zone, media, and the quantity of top picks. Thus, the positioning of the highlights considered has a significant impact on the after effects of the characterization procedure. Not the majority of the highlights are quantitative; some are subjective and require human intercession to decide their significance as for the general objective. This mediation happens just once all the while. We depend on a human master to create a judgment framework concerning the significance of each component. Condition 10 shows the type of the judgment grid.[3]

We utilize a pairwise correlation strategy to decide the overall significance of the removed highlights. This correlation procedure produces an estimation size of relative needs or loads of those highlights. The pairwise examinations are made by proportions of significance (of looked at highlights), which are assessed on a numeric scale from 1 to 9 .

The pairwise examinations are performed at the component level, where they are analyzed, two at once, 
regarding the general objective of the positioning procedure. The consequences of pairwise comparisons are gone into a network.. An element is similarly significant when contrasted with itself, along these lines, the corner to corner of the network must comprise of "1"values. What's more, the lattice must fulfill the connection fij $=1 /$ fji. The framework is perused as pursues: for instance, in the event that fij $=5$, at that point include $\mathrm{I}$ is of fundamental and solid significance over element $\mathrm{j}$. This additionally suggests the worth fji will be equivalent to $1 / 5$. This lattice is the essential unit of the examination and is additionally called "the pairwise correlation framework."[5]

After all the pairwise examination esteems are gone into the grid, a vector of needs is figured for the highlights. In numerical terms, the head eigenvector (the eigenvector related with the greatest eigen esteem) is acquired. We standardize the framework by partitioning every component of the grid by the aggregate of its section components, and this turns into the vector of needs. Calculation 2 demonstrates the calculation of the need vector. Given a lot of characteristics, $\mathrm{f}=\mathrm{fl}, \mathrm{f} 2 \ldots \mathrm{fn}$, where $\mathrm{n}$ is the quantity of highlights, the expert over and over thinks about one component to another until all conceivable pairwise correlations are finished.

A reliable and sound master ought to have the option to decide his pairwise inclinations, for example fij= vi/vj $\mathrm{i}$, $\mathrm{j}$, exactly. Along these lines, let us think about the results of this condition being fulfilled on the sections in the pairwise examination network. On the off chance that we compose fijfjk and utilize the condition $f_{i j}=v i / v \mathrm{j} \forall i, j$ then we can determine the accompanying:

$f_{i j} f_{j k}=\frac{v_{i}}{v_{j}} \frac{v_{j}}{v_{k}}=\frac{v_{i}}{v_{k}}=f_{i k}$

\begin{tabular}{|l|l|}
\hline Machine Learning Algorithm & Accuracy \\
\hline Decision Tree Classifier & 0.7579 \\
\hline Feature Ranking Algorithm & 0.7855 \\
\hline
\end{tabular}

Table 1: Accuracy of the Three Classification Algorithms

\section{Results and Discussion}

Dataset utilized for this comprised of almost 14640 tweets. In which positive tweets are 2364, negative tweets are 9176 and impartial tweets are 3100. In this test, 3 situations of testing procedure are completed as pursue:

1. Choice Tree Testing utilizing Airline Sentiment dataset.

2. Highlight Ranking Testing utilizing Airline Sentiment dataset.

3. Choice Tree Testing and Feature Ranking Testing employments in Airline sentiment dataset.

In the first testing procedure, the investigation of the application is performed. In the second testing procedure the examination of the information qualities is reasonable to be executed by utilizing the strategy for Feature Ranking, at that point it is performed. In the third testing procedure relative examination of the Decision Tree and Feature Ranking are performed.

Table 1 shows the classification results of two machine learning algorithms used: Decision Tree Classifier and Feature Ranking Algorithm. In these results Feature Ranking Algorithm showed better performance over Decision Tree Classifier.

\section{Conclusion}

To acquire a superior comprehension of how to evaluate data validity on Twitter, we quantified and described the substance and wellsprings of Twitter tweets. We gathered information which comprises of 14640 tweets. In light of our element extraction process, we planned a robotized order framework that comprises of four fundamental segments: a notoriety based part, a believability classifier motor, a client experience segment, and a component rank calculation. The notoriety based system channels dismissed data before beginning the appraisal procedure. The classifier motor part recognizes valid and non- dependable substance. The client aptitude segment yields appraisals of Twitter-client mastery on a particular subject. At last, the component rank calculation helps in choosing the best highlights, in light of the general significance of each element. The viability of the framework was assessed utilizing testing the dataset.

\section{References}

[1] A. Gupta, p. Kumaraguru, c. Castillo, and p. Meier, "tweetcred: real-time credibility assessment of content on twitter," in social informatics, ed: springer, 2014, pp. 228-243.

[2] C. Castillo, m. Mendoza, and b. Poblete, "information credibility on twitter," presented at the proceedings of the 20th international conference on world wide web, Hyderabad, India, 2011.

[3] D. Saez-trumper, "fake tweet buster: a webtool to identify users promoting fake news on twitter," 2014.

[4] M. Abdul-mageed, m. Diab, and s. Kübler, "samar: subjectivity and sentiment analysis for arabic social media," computer speech \& language, vol. 28, pp. 20- 37, 2014. 
[5] T. Mitra and e. Gilbert, "credbank: a large-scale social media corpus with associated credibility annotations," in ninth international aaai conference on web and social media, 2015.

[6] Y. Ikegami, k. Kawai, y. Namihira, and s. Tsuruta, "topic and opinion classification based information credibility analysis on twitter," in systems, man, and cybernetics (smc), 2013 ieee international conference on, 2013, pp. 4676-4681. 